\title{
Communicating hands: ERPs elicited by meaningful symbolic hand postures.
}

\author{
Gunter, TC
}

http://hdl.handle.net/10026.1/1021

10.1016/j.neulet.2004.09.011

Neurosci Lett

All content in PEARL is protected by copyright law. Author manuscripts are made available in accordance with publisher policies. Please cite only the published version using the details provided on the item record or document. In the absence of an open licence (e.g. Creative Commons), permissions for further reuse of content should be sought from the publisher or author. 


\title{
Communicating hands: ERPs elicited by meaningful symbolic hand postures
}

\author{
Thomas C. Gunter ${ }^{\mathrm{a}, *}$, Patric Bach ${ }^{\mathrm{b}}$ \\ ${ }^{a}$ Max-Planck-Institute for Human Cognitive and Brain Sciences, PO Box 500355, D-04303 Leipzig, Germany \\ ${ }^{\mathrm{b}}$ Center for Cognitive Neuroscience, School of Psychology, University of Wales, Bangor, UK
}

Received 26 May 2004; received in revised form 2 September 2004; accepted 3 September 2004

\begin{abstract}
Meaningful and meaningless hand postures were presented to subjects who had to carry out a semantic discrimination task while electrical brain responses were recorded. Both meaningful and control sets of hand postures were matched as closely as possible. The ERPs elicited by meaningless hand postures showed an anteriorly distributed N300 and a centro-posteriorly distributed N400 component. The N300 probably reflects picture-specific processes, whereas the N400-effect probably reflects processing in an amodal semantic network. The scalpdistribution of the N400-effect, which is more posterior than usually reported in picture processing, suggests that the semantic representations of the concepts expressed by meaningful hand postures have similar properties to those of abstract words.
\end{abstract}

(c) 2004 Elsevier Ireland Ltd. All rights reserved.

Keywords: Hand postures; Gestures; Emblems; Nonverbal communication; N300; N400

Face-to-face communication relies on visual and auditory cues. The auditory stream has the highest information content, and is therefore investigated in most language research. Visual information (i.e., gestures or lip movements), however, also plays an important, though less explored, role in normal communication [6]. Gestures, for instance, can assist the processing of auditory information through disambiguation $[8,11]$. Hand postures, which are a specific type of gesture, can have quite clear-cut meanings, as in for example the 'thumbs-up' gesture. Such meaningful hand postures, or 'emblems' [16], are fascinating communicative tools because a very complex message can be transferred with the use of a single gesture. Emblems can be considered to be unspoken words or phrases, in that they are used symbolically and are theorized to be lexicalized [16], i.e., an emblem may have a mental lexicon entry. It is, therefore, important to find experimental evidence for whether or not the semantic processing of emblems is similar to that of words. Note that the use of

\footnotetext{
* Corresponding author. Tel.: +49 341 9940115; fax: +49 3419940113. E-mail address: Gunter@cbs.mpg.de (T.C.Gunter).
}

emblems has a different linguistic status than the hand postures used in sign language, which can also reflect syntactic [14] and morphological [20] information.

In ERP research, lexical-semantic processes are found to be reflected in a centro-parietal negativity at around $400 \mathrm{~ms}$ (cf. N400) after stimulus onset in both spoken and written language [13] as well as in American Sign Language [18]. The N400 is large whenever a word does not fit a context and is reduced in amplitude whenever it does fit. Pseudo-words elicit a large N400 compared to words [3,4]. The scalp distribution of the $\mathrm{N} 400$ found in sentence processing is influenced by the concreteness of the words. Concrete words, or words referring to pictureable objects, show a more anterior distribution than abstract words, which elicit a more centro-parietally distributed N400 [10]. Experiments looking at the semantic processing of anomalous pictures presented at a sentencefinal position report a frontally distributed N300 component and an anteriorly distributed N400 [7].

In contrast to the N400 component, the N300 appears to be specific for picture processing $[2,7,17]$. In picture identification tasks, the N300 reflects picture-specific semantic processing but not the processing of the physical properties 


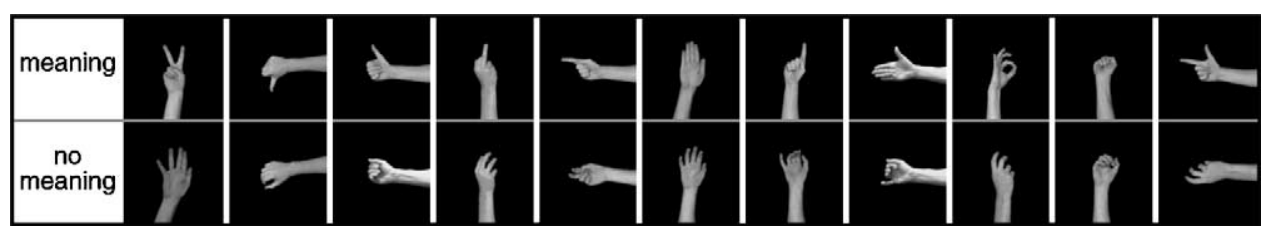

Fig. 1. The 11 hand postures used in the experiment. For each hand posture-pair, the meaningful one (or emblem) is presented in the upper part of the figure, while the matching meaningless hand posture is presented in the lower part. Both types of hand postures were matched in shape as closely as possible.

of the pictures $[2,17]$. In an experiment that explored picture stories, the N300 was suggested to reflect a nonverbal semantic mechanism, namely the activation of image-based representations [21]. Recently, an experiment using a wordpriming paradigm suggested that the N300 reflects an early semantic categorization of object stimuli into members of a semantically meaningful group [9].

The present experiment explores the underlying brain mechanisms elicited during the semantic processing of emblems. Meaningful and meaningless hand postures are compared with each other in a semantic categorization task. Because of the pictorial nature of the hand postures and the necessity to semantically categorize the stimuli into meaningful and meaningless postures, we expect an N300-effect for the meaningless hand postures when compared to meaningful ones. Since emblems do not semantically refer to concrete objects, they will induce abstract semantic processing. Therefore, we hypothesized that a classical, centro-parietally distributed, N400-effect would be elicited when meaningless hand postures are compared to emblems.

Twenty-two native German-speaking students (11 females, 21-30 years (mean 24.2 years), right-handed, normal or corrected-to-normal vision) were paid 25 Euros for their participation. All participants signed a written informed consent.

Participants were presented with meaningful and meaningless hand postures that had been rated in a separate study by a group of 47 German native speakers and were classified as meaningful or meaningless by at least $80 \%$ of the raters (this resulted in 11 test-control pairs; see Fig. 1). The meaningful postures were judged on their meaning as well. Generally, meanings listed for emblems clustered around one meaning. Three emblems, however, were less clear-cut: emblem 1 (53\% 'victory', 32\% 'peace', $15 \%$ other responses); emblem 7 (48\% 'attention', 45\% 'look above', 7\% other); and emblem 11 (58\% 'pistol', 29\% 'two' and 13\% other). Both the meaningful and control postures for each pair had approximately the same shape.

The stimulus set consisted of digitized gray-scale photos $(400 \times 400$ pixels) of 66 meaningful and 66 meaningless hand postures (i.e., the 11 posture-pairs each photographed using the hands of six different people). Out of this stimulus set, the experimental set of 198 meaningful and 198 meaningless hand postures was created. These 396 photos were randomly presented in a discrimination task. One trial consisted of the following events: (1) a picture of a hand posture appeared for $700 \mathrm{~ms}$, (2) a blank screen was presented for $500 \mathrm{~ms}$, (3) a GO signal appeared, which indicated that the participant had to judge whether a hand posture was meaningful or meaningless with a button press. Immediately after the response, the next trial started. Each hand posture was repeated three times in the complete experiment of 396 total trials.

The discrimination task typically lasted $25 \mathrm{~min}$, during which the participants were seated in a dimly lit, sound-proof cabin, facing a color video screen at a distance of $110 \mathrm{~cm}$. Before the experimental task, they performed a short training session of 24 trials.

The electroencephalogram (EEG) was recorded with 59 $\mathrm{Ag}-\mathrm{AgCl}$ electrodes (electrocap) from $\mathrm{Fp} 1, \mathrm{FpZ}, \mathrm{Fp} 2$, Af7, Af3, AfZ, Af4, Af8, F7, F5, F3, Fz, F4, F6, F8, Ft7, Fc5, Fc3, Fcz, Fc4, Fc6, Ft8, Ft7, Fc5, Fc3, Fcz, Fc4, Fc6, Ft8, T7, C5, C3, Cz, C4, C6, T8, Tp7, Cp5, Cp3, CpZ, Cp4, Cp6, Tp8, P7, P5, P3, Pz, P4, P6, P8, Po7, Po3, Poz, Po4, Po8, O1, $\mathrm{Oz}, \mathrm{O} 1$ and right mastoid, each referred to the left mastoid. Bipolar horizontal and vertical EOGs were recorded for artifact rejection purposes. Electrode resistance was kept under $5 \mathrm{k} \Omega$. The signals were recorded continuously with a band pass between dc and $70 \mathrm{~Hz}$ and digitized at a rate of $250 \mathrm{~Hz}$.

Average ERPs, starting $200 \mathrm{~ms}$ before and lasting $1000 \mathrm{~ms}$ after the presentation of the pictures, were computed for each electrode position and each of the two conditions. Only correctly discriminated hand postures (i.e., 99\%) entered the analysis. Due to ocular artifacts, an additional $6.6 \%$ of the trials were excluded. Averages were aligned to a $200 \mathrm{~ms}$ prestimulus baseline. Statistical analyses were performed using five anterior and five posterior Regions of Interest (ROIs; see Fig. 2b) in the N300 and N400 latency windows (300-400 ms and $450-550 \mathrm{~ms}$, respectively). The within-subject factors for the repeated measure ANOVAs were meaning (meaningful versus meaningless), anterior/posterior (2) and ROI (5). Reported scalp-distribution effects were always additionally checked and found significant using the method of McCarthy and Wood [15].

As can be seen in Fig. 2a and b, meaningless compared to meaningful hand postures gave rise to a right-frontally distributed negativity that continued during the time in which a centro-parietal N400 occurred.

The 300-400-ms time window revealed an interaction between the factors of meaning, anterior/posterior, and ROI $(F(4,84)=3.52, p<0.033, \varepsilon=0.55)$. On the basis of this threeway interaction, a step-down analysis was carried out which tested the anterior ROIs separately because visual inspection of the maps showed that the negativity has its maximum at anterior electrodes. The analysis on the anterior ROIs showed 

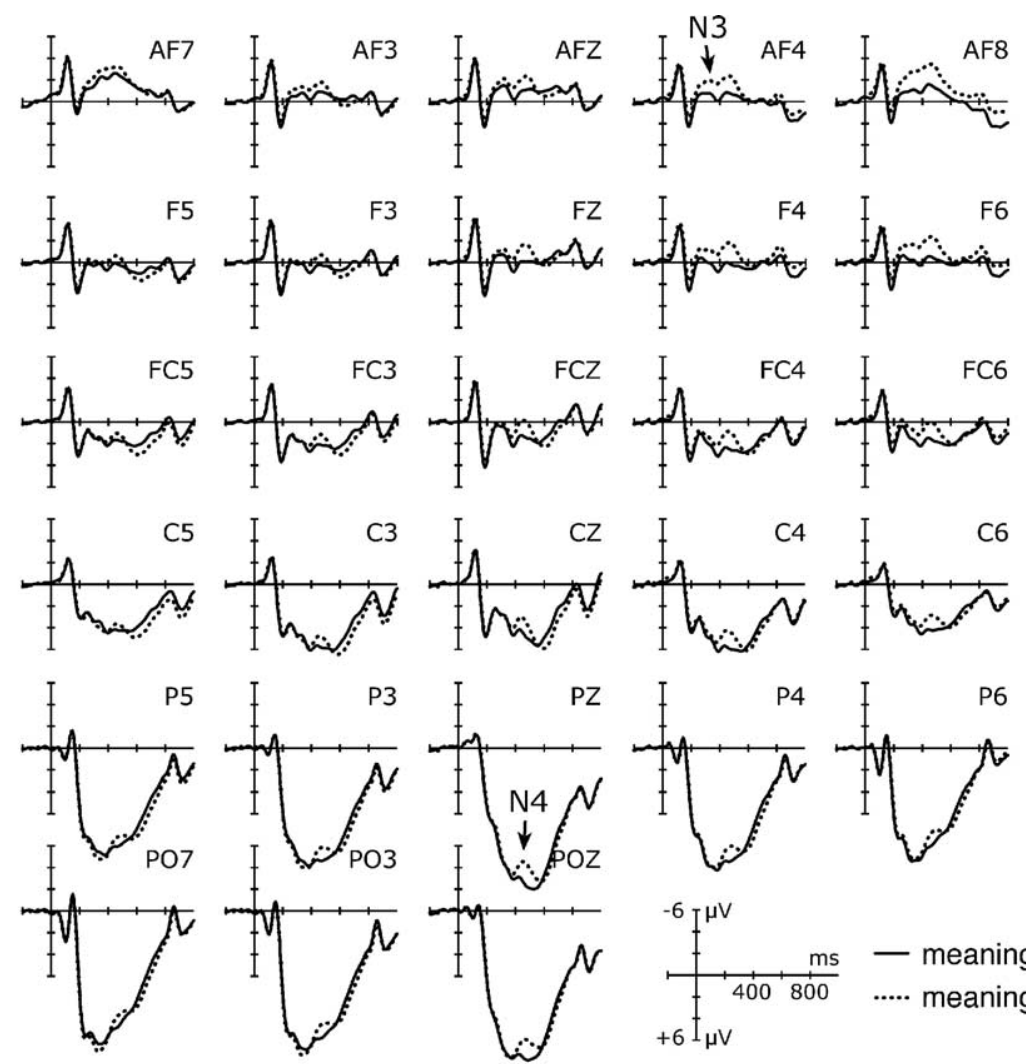

(a)

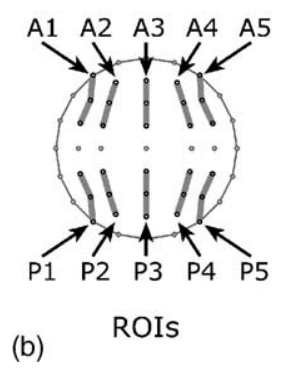

(c)

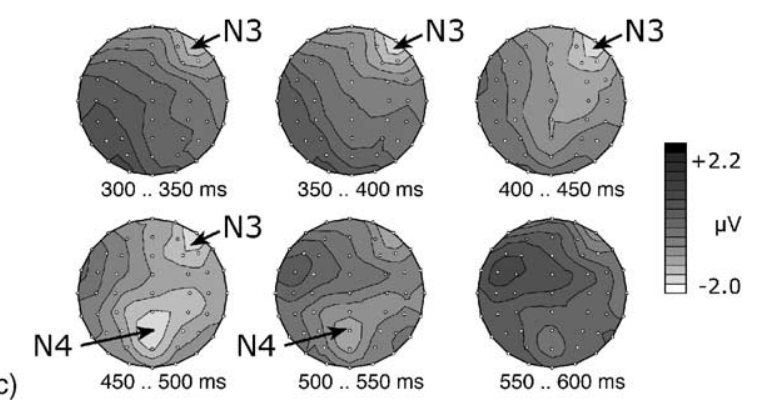

Fig. 2. The ERP-data for the meaningful vs. meaningless hand postures. (a) The ERPs elicited by both types of hand postures (18 selected electrodes). The solid line represents the ERP elicited from meaningful hand postures, the dotted line corresponds to the meaningless hand postures. The $x$-axis represents electrical activity in micro-volt (positive is plotted down). The $y$-axis represents time in milliseconds (each tic represents $200 \mathrm{~ms}$ ). (b) The Regions of Interest (ROI) used for the scalp distribution analysis. Note that the five anterior ROIs are labeled A1-A5, while the five posterior ROIs are labeled P1-P5. (c) Voltage maps of the difference between the ERPs of meaningful versus meaningless hand postures in $50 \mathrm{~ms}$ steps in the time window between 300 and $600 \mathrm{~ms}$. This is the window where the N300 transfers into the N400 time window.

a main effect for meaning $(F(1,21)=28.99, p<0.0001)$ and an interaction between meaning and $\mathrm{ROI}(F(4,84)=17.23, p$ $<0.0001, \varepsilon=0.51)$. In order to test the lateralization of the negativity, two new ROIs were computed, one for left anterior (A1, A2) and one for right anterior (A4, A5). An ANOVA with the factors meaning and lateralization (left versus right anterior ROIs) showed a main effect of meaning $(F(1,21)=$ 29.07, $p<0.0001)$ and an interaction between meaning and lateralization $(F(1,21)=24.44, p<0.0001)$. This interaction indicates that the negativity in the 300-400-ms time window is lateralized to the right and is therefore identified as an N300.
The 450-550-ms time window also showed an interaction between meaning, anterior/posterior, and ROI $(F(4,84)$ $=12.98, p<0.0001, \varepsilon=0.52$ ). Therefore, separate analyses were carried out on the anterior and posterior ROIs. The anterior analysis showed a main effect of meaning $(F(1,21)=$ $8.38, p<0.0087)$ and an interaction of meaning with ROI $(F(4,84)=9.61, p<0.0002, \varepsilon=0.558)$. The posterior analysis showed main effects of meaning $(F(1,21)=8.38, p<$ $0.0087)$ and $\operatorname{ROI}(F(4,84)=11.02, p<0.0005, \varepsilon=0.39)$ and an interaction between meaning and ROI $(F(4,84)=5.47$, $p<0.0058, \varepsilon=0.554)$. On the basis of the interactions between meaning and ROI, we were licensed to investigate the 
Table 1

ERP effect size (i.e., meaningless minus meaningfull) in microvolt as found in the 450-550-ms time interval for all anterior (A1-A5) and posterior (P1-P5) ROIs

\begin{tabular}{llc}
\hline ROI & Anterior & Posterior \\
\hline 1 & -0.29 & -0.67 \\
2 & -0.41 & -0.95 \\
3 & -0.79 & -1.51 \\
4 & -1.10 & -1.04 \\
5 & -1.32 & -0.87 \\
\hline
\end{tabular}

The posterior ROIs show their maximum at the central ROI (i.e., the N400) whereas the anterior ROIs show their maximum at the most right ROI (i.e., the N300).

lateralization of the effects at anterior and posterior ROIs. As in the N300 latency window, new ROIs were defined: anterior left (A1, A2), anterior right (A4, A5), posterior left (P1, P2), and posterior right $(\mathrm{P} 4, \mathrm{P} 5)$.

The analyses of the posterior ROIs only showed a main effect of meaning $(F(1,21)=5.72, p<0.026)$ and did not show any interaction between meaning and lateralization $(F(1,21)$ $=0.51$, n.s.). This statistical analysis showed that there is a posterior component that has no lateralization. It was therefore identified as an N400 (see also Table 1).

The analyses of the anterior ROIs showed a main effect of meaning and an interaction between meaning and lateralization $(F(1,21)=8.87, p<0.0072 ; F(1,21)=16.36, p<$ $0.0006)$. These analyses show that there is a right anterior component (see also Table 1). A major question is whether this component is an extended N300 or not.

To explore whether the right frontal negativity is the same in both time windows, we carried out an analysis exclusively on the right frontal ROIs (A3, A4, and A5) with an additional variable called time-window (300-400 ms versus $450-550 \mathrm{~ms}$ ). This analysis showed a significant main effect of meaning $(F(1,21)=34.68, p<0.0001)$ and an interaction between meaning and ROI $(F(2,42)=11.44, p<0.0006, \varepsilon=$ 0.73 ) only. Most importantly, no main effect of time window or any interaction with time window was found. This indicates that the right frontal negativity in both time windows is probably (but not compulsory) the same.

In summary, in comparison to emblems, the meaningless hand postures show an N300 that extends into the time window where an N400 is elicited (see Fig. 2c).

The present experiment compared the processing of meaningful emblems versus meaningless hand postures and explored whether emblem processing resembles normal word processing. Because emblems are theorized to be lexicalized, this comparison is analogous to word versus pseudoword processing. Note that the N300- and N400-effect were relatively small. This may have been due to the paradigm used: on the one hand, it is possible that due to the large amount of repetition, the effects were reduced, and on the other hand, a more general paradigm like priming probably would have facilitated the ERP effects [2].

Compared to emblems, the meaningless hand postures showed a right frontal N300 and a centro-parietal N400. The
N300-effect shows similarity with what is known about the semantic processing of pictures $[2,7,17,21]$. The N300 possibly reflects the activation of an image-based representation of the emblems, perhaps by means of an early semantic categorization of the hand postures.

Emblems showed a reduced N400 compared to meaningless hand postures. Thus, they elicit an N400-effect that shows similarities to that elicited by words when compared to psuedowords $[3,4]$. The posterior scalp distribution of the N400effect that was obtained was expected, as the emblems used had abstract meanings. This finding makes it plausible that at least some aspects of the semantic processes underlying emblem processing are similar to those underlying abstract word processing. That is, if the N400-effect reflects processing in a common amodal semantic network [19], one could suggest that emblems and words share a common type of semantic representation [1,5]. Note, however, that the earlier N300effect found in emblem processing suggests that emblems may access a separate picture-related semantic network [9] in addition to an amodal one. The present data therefore suggest that emblems have a kind of pictorial representation not present while processing written words. Some theories on the ontogenesis of gestures suggest that in many cases, emblems began as iconic gestures that were ritualized and stabilized as part of a gesture code [12]. A residual pictorial component during emblem processing is therefore not implausible.

The duration of the N300-effect for emblems, however, was surprising. It was still present during the elicitation of the N400-effect and did not disappear as could have been expected on the basis of the literature on semantic picture processing [21]. One possibility is that this prolonged N300-effect is specific for emblems and does not show up during picture processing. If the prolonged N300 is specific for emblems, it might reflect either that image-based representations arising from emblems are active especially long, or that the categorization process that had to be carried out during the present experiment was relatively difficult. Note however, that studies exploring semantic picture processing typically use pictures that depict objects or have a concrete meaning. Such studies therefore have difficulty separating the frontal $\mathrm{N} 300$ from the anteriorly elicited N400 due to component overlap. Thus, it is possible that in such studies, ongoing N300 activity is overlapped (and therefore obscured) by an anterior N400. This possibility is intriguing because it suggests that picture-specific processing, i.e., the access of image-based representations [21], continues even after amodal semantics has been accessed. Further experiments are clearly needed to explore these issues further.

The present study showed that although meaningful hand postures, or emblems, are much more pictorial in nature than words, they may be processed by a similar semantic system as evidenced by the N400-effect obtained during emblem processing. 


\section{Acknowledgments}

We wish to thank Sven Gutekunst for his technical support; Ina Koch for the data acquisition; and Akinori Nakamura for preparing the stimuli and helpful discussion. We are indebted to Kerrie Elston-Güttler for helpful comments on earlier drafts of this manuscript.

\section{References}

[1] J.R. Anderson, G.H. Bower, Human Associative Memory, Wiley, New York, 1973.

[2] S.E. Barrett, M.D. Rugg, Event-related potentials and the semantic matching of pictures, Brain Cogn. 14 (1990) 201-212.

[3] S. Bentin, Visual word perception and semantic processing-an electrophysiological perspective, Israel J. Med. Sci. 23 (1987) 138-144.

[4] S. Bentin, G. McCarthy, C.C. Wood, Event-related potentials, lexical decision and semantic priming, Electroencephalogr. Clin. Neurophysiol. 60 (1985) 343-355.

[5] A. Caramazza, A.E. Hillis, B.C. Rapp, C. Romani, The multiple semantics hypothesis-multiple confusions, Cogn. Neuropsychol. 7 (1990) 161-189.

[6] H.H. Clark, Using Language, Cambridge University Press, Cambridge, GB, 1996.

[7] K.D. Federmeier, M. Kutas, Meaning and modality: influences of context, semantic memory organization, and perceptual predictability on picture processing, J. Exp. Psychol. Learn. Mem. Cogn. 27 (2001) 202-224.

[8] S. Goldin-Meadow, The role of gesture in communication and thinking, Trends Cogn. Sci. 3 (1999) 419-429.

[9] J.P. Hamm, B.W. Johnson, I.J. Kirk, Comparison of the N300 and N400 ERPs to picture stimuli in congruent and incongruent con- texts, Electroencephalogr. Clin. Neurophysiol. 113 (2002) 13391350.

[10] P.J. Holcomb, J. Kounios, J.E. Anderson, W.C. West, Dual-coding, context-availability, and concreteness effects in sentence comprehension: an electrophysiological investigation, J. Exp. Psychol. Learn. Mem. Cogn. 25 (1999) 721-742.

[11] S.D. Kelly, D.J. Barr, R.B. Church, K. Lynch, Offering a hand to pragmatic understanding: the role of speech and gesture in comprehension and memory, J. Mem. Lang. 40 (1999) 577-592.

[12] A. Kendon, Gestures, their origins and distribution, Semiotica 37 (1981) 129-163.

[13] M. Kutas, K.D. Federmeier, Electrophysiology reveals semantic memory use in language comprehension, Trends Cogn. Sci. 4 (2000) 463-470.

[14] S. Lidell, American Sign Language Syntax, Mouton, 1980.

[15] G. McCarthy, C.C. Wood, Scalp distributions of event-related potentials - an ambiguity associated with analysis of variance models, Electroencephalogr. Clin. Neurophysiol. 62 (1985) 203-208.

[16] D. McNeill, Hand and Mind: What Gestures Reveal About Thought, University of Chicago Press, Chicago, 1992, pp. 56-62.

[17] W.B. McPherson, P.J. Holcomb, An electrophysiological investigation of semantic priming with pictures of real objects, Psychophysiology 38 (1999) 53-65.

[18] H.J. Neville, S.A. Coffey, D.S. Lawson, A. Fischer, K. Emmorey, U. Bellugi, Neural systems mediating American sign language: effects of sensory experience and age of acquisition, Brain Lang. 57 (1997) 285-308.

[19] A. Nigam, J.E. Hoffman, R.F. Simons, N400 to semantically anomalous pictures and words, J. Cogn. Neurosci. 4 (1992) 15-22.

[20] T. Supalla, The classifier system in American sign language, in: C. Graig (Ed.), Noun Classes and Categorization, John Benjamins, 1986, pp. 181-215.

[21] W.C. West, P.J. Holcomb, Event-related potentials during discourselevel semantic integration of complex pictures, Cogn. Brain Res. 13 (2002) 363-375. 